\title{
Different Contributions of Microtubule Dynamics and Transport to the Growth of Axons and Collateral Sprouts
}

\author{
Gianluca Gallo and Paul C. Letourneau \\ University of Minnesota, Department of Cell Biology and Neuroanatomy, Minneapolis, Minnesota 55455
}

\begin{abstract}
Axonal growth is believed to depend on microtubule transport and microtubule dynamic instability. We now report that the growth of axon collateral branches can occur independent of microtubule dynamic instability and can rely mostly on the transport of preassembled polymer. Raising embryonic sensory neurons in concentrations of either taxol or nocodazole (NOC) that largely inhibit microtubule dynamics significantly inhibited growth of main axonal shafts but had only minor effects on collateral branch growth. The collaterals of axons raised in taxol or nocodazole often contained single microtubules with both ends clearly visible within the collateral branch ("floating" microtubules), which we interpret as microtubules undergoing transport. Furthermore, in these collaterals there was a distoproximal gradient in microtubule mass, indicating the distal accumulation of transported polymer. Treatment of cultures
\end{abstract}

Axons reach their target tissues through growth cone navigation and the establishment of axon collateral branches. The formation of axon collaterals is of fundamental importance to the development of innervation patterns within target tissues (O'Leary and Terashima, 1988; Heffner et al., 1990; Bhide and Frost, 1991; O'Leary et al., 1991; Ghosh and Shatz, 1992; Kadhim et al., 1993; O'Leary and Koester, 1993; Kennedy and Tessier-Lavigne, 1995; Ozaki and Snider, 1997) and is regulated by both positive and inhibitory extrinsic cues (Zhang et al., 1994; Kennedy and Tessier-Lavigne, 1995; Schwegler et al., 1995; Castellani et al., 1998; Gallo and Letourneau, 1998). Collaterals are initiated as filopodial sprouts from axonal shafts (Sato et al., 1994; Yu et al., 1994; Bastmeyer and O'Leary, 1996; Gallo and Letourneau, 1998; Szebenyi et al., 1998) that subsequently mature into branches capable of responding to extracellular guidance cues (Gallo and Letourneau, 1998). Although much has been learned about the cytoskeletal rearrangements that underlie growth of the main axon, relatively little is known about the cytoskeletal mechanisms that produce collateral branches [but see Yu et al. (1994)]. In an effort to elucidate the cytoskeletal mechanisms responsible for collateral branch formation, we investigated the roles of actin filaments and microtubule dynamics and transport in the generation of axon collaterals by chick embryonic sensory axons in vitro.

Microtubule mass could be added to the axonal array by two

Received Oct. 1, 1998; revised Jan. 21, 1999; accepted Feb. 25, 1999.

This research was supported by National Institutes of Health Grant HD 19950-12 (P.C.L.). We thank Mr. G. Service (University of Minnesota) for technical assistance.

Correspondence should be addressed to Dr. Gianluca Gallo, Department of Cell Biology and Neuroanatomy, University of Minnesota, 4-144 Jackson Hall, 321 Church Street SE, Minneapolis, MN 55455.

Copyright (C) 1999 Society for Neuroscience 0270-6474/99/193860-14\$05.00/0 with a high dose of nocodazole to deplete microtubules from collaterals, followed by treatment with 4-20 nм vinblastine to inhibit microtubule repolymerization, resulted in the timedependent reappearance and subsequent distal accumulation of floating microtubules in collaterals, providing further evidence for microtubule transport into collateral branches. Our data show that, surprisingly, the contribution of microtubule dynamics to collateral branch growth is minor compared with the important role of microtubule dynamics in growth cone migration, and they indicate that the transport of microtubules may provide sufficient cytoskeletal material for the initial growth of collateral branches.

Key words: microtubule; filopodium; axon; collateral; $d y-$ namic instability; transport mechanisms: (1) polymerization of tubulin subunits at the plus ends of axonal microtubules and (2) the transport of preassembled polymer. These two mechanisms are not mutually exclusive, and it has been argued that both contribute to axonal growth (Black, 1994; Baas, 1997). The growth and guidance of the main axon have been shown to depend in part on the dynamic instability of the plus ends of microtubules (Tanaka et al., 1995; Rochlin et al., 1996; Williamson et al., 1996; Yu and Baas, 1995; Challacombe et al., 1997; Gallo, 1998). Although controversy exists about the role and magnitude of microtubule transport in the growth of neuronal process [for opposing viewpoints, see the reviews by Baas (1997) and Joshi (1998)], several lines of evidence demonstrate that at least some microtubules undergo centrif ugal transport from the soma and contribute to the axonal microtubule array (Reinsch et al., 1991; Okabe and Hirokawa, 1992; Baas and Ahmad, 1993; Smith, 1994; Ahmad and Baas, 1995; Terasaki et al., 1995; Yu et al., 1996; Slaughter et al., 1997; Ahmad et al., 1998). At present, little information is available on the relative contributions of the dynamic changes of microtubule plus ends and microtubule transport in the generation and growth of axon collaterals.

We now report that the main axons and axon collaterals of embryonic dorsal root ganglion (DRG) neurons exhibit different sensitivities to treatments that inhibit the dynamic growth of microtubule plus ends. Furthermore, we provide evidence for the transport of microtubules from the main axon into collateral branches and show that the growth of microtubule plus ends by tubulin polymerization also contributes to the microtubule array of axon collateral branches. Therefore, as for axonal growth, both the dynamics and transport of microtubules contribute to axon collateral growth. However, unlike axons, elongation of collateral branches is relatively normal under conditions that greatly atten- 
uate microtubule dynamics, indicating that the transport of microtubules may be the most important mechanism of microtubule advance in the initial formation of collateral branches.

\section{MATERIALS AND METHODS}

Culturing of embryonic DRG neurons. Cell culture was performed as described previously (Gallo et al., 1997; Gallo and Letourneau, 1998). Briefly, lumbosacral embryonic day (E) 9-10 chick DRG neurons were chemically and mechanically dissociated and cultured in F12 serum-free medium (Life Technologies, Grand Island, NY) supplemented with additives and $2 \mathrm{ng} / \mathrm{ml}$ NGF (R\&D systems, Minneapolis, MN). DRG neurons were plated at densities of $0.5-1.0$ ganglia per coverslip. For immunocytochemistry, neurons were raised on heat-treated $18 \times 18 \mathrm{~mm}$ glass coverslips, whereas for video observation, $24 \times 30 \mathrm{~mm}$ heat-treated glass coverslips were mounted on a $22 \mathrm{~mm}$ hole drilled into the bottom of a $35 \mathrm{~mm}$ tissue culture dish. For biochemical analysis of tubulin levels, purified neurons (preplated for $2 \mathrm{hr}$ on fibronectin-coated tissue-culture plastic dishes) were raised overnight in $35 \mathrm{~mm}$ tissue-culture plastic dishes. Culturing substrata were coated overnight with $50 \mu \mathrm{g} / \mathrm{ml} \mathrm{fi-}$ bronectin (Life Technologies). All experiments were performed using 20- to 36-hr-old cultures.

Immunocytochemistry. To study the cytoskeleton of collateral branches, cultures were simultaneously fixed and extracted in cytoskeletal buffer [prepared as described in Gallo (1998)] with $0.2 \%$ glutaraldehyde and $0.1 \%$ Triton $\mathrm{X}-100$ for $15 \mathrm{~min}$, followed by a $15 \mathrm{~min}$ incubation in $1 \mathrm{mg} / \mathrm{ml}$ sodium borohydride in $\mathrm{Ca}^{2+}-\mathrm{Mg}^{2+}$-free PBS (CMFPBS), $\mathrm{pH}$ 7.1. Cultures were then washed three times with CMFPBS and incubated with primary antibodies in blocking solution (1\% fish gelatin in CMFPBS) for $45 \mathrm{~min}$. To visualize total microtubules we used an anti- $\beta$-tubulin monoclonal antibody (Amersham, Arlington Heights, IL) at 1:100. Detyrosinated $\alpha$-tubulin was labeled with a rabbit polyclonal antibody that was kindly provided by Dr. G. Gundersen (Columbia University, New York, NY), at 1:800. Cultures were then washed three times with CMFPBS and incubated for 45 min with the appropriate fluorescent secondary antibody and $8 \mu \mathrm{l} / 100 \mu \mathrm{l}$ of a rhodamine-phalloidin (Molecular Probes, Eugene, OR) stock solution prepared in methanol. Fluorescein-conjugated goat anti-mouse (Cappel, Durham, NC) and rhodamine-conjugated goat anti-rabbit (Cappel) secondary antibodies, both used at 1:400 in blocking solution, were used to detect the $\beta$-tubulin and detyrosinated $\alpha$-tubulin primary antibodies, respectively. Cultures were then washed twice in CMFPBS and once in distilled water before mounting in media containing $10 \mathrm{mg} / \mathrm{ml}$ $p$-phenylenediamine (Sigma, St. Louis, MO) on glass coverslips.

Pharmacological treatments. Taxol (7 nM; Natural Products Branch, National Cancer Institute, Bethesda, MD) or nocodazole (NOC) (83 nM; Sigma) were added to DRG cells at the time of plating. In experiments using nocodazole $(2 \mu \mathrm{g} / \mathrm{ml})$ to depolymerize microtubules, the drug was added acutely to the cultures. In some experiments, after an initial treatment, nocodazole was replaced with vinblastine $(4-20 \mathrm{~nm}$; Sigma). To prevent microtubules from undergoing repolymerization during the drug change, the nocodazole-containing medium was removed and immediately replaced with $1 \mathrm{ml}$ of prewarmed $\left(40^{\circ}\right)$ medium containing the desired concentration of vinblastine. After 1-2 min, the medium was again exchanged with $1 \mathrm{ml}$ of prewarmed medium containing vinblastine. The cultures were then returned to the incubator. In control experiments, medium containing DMSO (1:500), the vehicle for all drugs used in the present studies, was used to replace the nocodazole-containing medium.

Data collection and analysis. The behavior of living axons and established collaterals was monitored by phase-contrast optics using an inverted microscope (IM-35, Zeiss, Thornwood, NY) equipped with a Newvicon video camera (NC-65, Dage-MTI, Michigan City, IN) for periods of $1 \mathrm{hr}$. Image acquisition and enhancement (each image was the average of 16 frames obtained over $0.5 \mathrm{sec}$ ) were performed using IMAGE 1 software (Universal Imaging, West Chester, PA) running on a 486/33 MHz computer (Gateway 2000, North Sioux City, SD). Images were stored on optical disks using an OMDR (TQ-3038F, Panasonic Industrial, Secaucus, NJ). For the purpose of studying axonal filopodial formation rates, records were made with an interframe interval of 0.5 min. Records of collateral or axonal growth were made using an interframe interval of $1 \mathrm{~min}$.

Established collaterals were identified on the basis of morphological criteria (Sato et al., 1994; Yu et al., 1994; Bastmeyer and O'Leary, 1996; Gallo and Letourneau, 1998). To be classified as a collateral, a neuronal process had to meet all of the following requirements: (1) the origin must be from the main axon, (2) the width of the process must be smaller than that of the axon, (3) the angle at which the process extends from the main axon must be $>60^{\circ}$ (defined as the degree of angular displacement of the collateral from the distal, growth cone-bearing portion of the axon), and (4) the length of the collateral must be no more than $50 \%$ of the length of the axon between the point of origin of the collateral and the growth cone. Criteria (2) to (4) are intended to eliminate from consideration as collaterals axonal branches produced by growth cone bifurcation. DRG neurites that are formed by growth cone bifurcation exhibit similar axonal widths, extend at an angle of $\sim 60 \%$ from one another, giving the appearance of a Y, and extend with similar growth rates [Gallo, personal observations; see Letourneau et al. (1986) for a detailed study of DRG growth cone bifurcation]. In support of the notion that these are valid criteria for defining axon collaterals, the data in Table 2 demonstrate that these criteria yielded two distinctly different populations of neuritic processes in terms of their dynamics and rates of growth.

Video recordings of axonal or collateral growth were used to measure hourly growth rates and time periods that processes spent growing, retracting, or being quiescent ( $1 \mathrm{hr}$ recording period). All determinations of axonal or collateral tip movement were made from the distal-most phase dark extent of the process. The hourly growth rate was obtained by measuring the distance between the tip of the process at the start and the end of a $1 \mathrm{hr}$ video recording. The behavior of a process during a $5 \mathrm{~min}$ interval was scored as growing if the tip extended distally, retracting if the tip receded proximally, or as quiescent if no displacement occurred. Lateral displacements of processes without obvious change in length were scored as quiescence. Furthermore, it must be noted that these determinations do not include growth cone lamellipodial or filopodial activity but are limited to the displacement of the process tip.

To quantify aspects of cytoskeletal arrangements during collateral formation (e.g., frequency of microtubule invasion of sprouts), we used a Zeiss epifluorescence microscope $(400 \times$ final magnification) equipped with a calibrated grid eye piece that allowed us to determine the approximate length of collateral sprouts. A two-step scoring procedure was used to determine microtubule invasion of collaterals: (1) sprouts were identified by their cortical rhodamine-phalloidin staining, and then (2) the fluorescently labeled microtubules were viewed to determine whether microtubules were present within identified sprouts. F-actin- and $\beta$-tubulin-stained cultures were also used to determine the frequency of collateral sprouts along axons by visualizing the actin staining alone. On occasion, sprouts had undergone branching and the length of the sprout was determined from the apparent longest "branchlet." Cultures stained for $\beta$-tubulin and detyrosinated $\alpha$-tubulin were similarly scored to determine whether the microtubule(s) extending from the microtubule array of the main axon into collaterals exhibited only $\beta$-tubulin staining or was double-stained for both tubulin types. Because in cultures stained for $\mathrm{F}$-actin and $\beta$-tubulin we never observed microtubules extending from the axonal array that were not contained by a phalloidin-stained cortex, we are confident that all microtubules scored in the double microtubule-stained cultures were microtubules contained in collaterals. A two-step scoring procedure was used to determine whether microtubules in collaterals exhibited labeling for both $\beta$ - and detyrosinated $\alpha$-tubulin isoforms, labeled as described above (see Immunocytochemistry). (1) Microtubules were first identified using the fluorescein filters to visualize $\beta$-tubulin, and then (2) we determined whether the same microtubules labeled for detyrosinated $\alpha$-tubulin by using the rhodamine filter. Bundles of microtubules were scored as positive if at least one microtubule exhibited detyrosinated $\alpha$-tubulin staining. Therefore, this scoring procedure yields the frequency with which collateral microtubule arrays contain at least one labeled microtubule. In cultures doublestained for $\beta$-tubulin and detyrosinated $\alpha$-tubulin, "floating" microtubules (see Results), single microtubules isolated from the rest of the microtubule array, were determined to be in sprouts by the faint background cytoplasmic staining that appears when staining with detyrosinated $\alpha$-tubulin antibodies. In all cases, only processes that were attached to the substratum along their whole length and did not appear to have been damaged during the immunocytochemical procedure were used for data collection. Data were collected from a minimum of three cultures in each treatment condition.

Microtubule length-width measurements were made using the length and intensity functions of NIH Image 1.55 (Bethesda, MD) on images obtained using a Nikon Diaphot 200 microscope (40× oil objective) equipped with a CCD camera (Paulteck Imaging, Nevada City, CA). All images were acquired using identical camera settings, and measurements were performed on unaltered raw images. Measurements of microtubule 
length were performed as follows: floating microtubules were measured from tip to tip, and the length of microtubules invading sprouts was measured from the point of origin from the axonal microtubule array to the tip of the microtubule(s). The length of microtubules that deviated from a straight line was determined by measuring, and subsequently adding, individual segments of the microtubules. Comparisons of the intensity profiles of the $\beta$-tubulin staining of floating microtubules and microtubules invading axonal filopodia with that of single microtubules in the lamellipodia of fibroblasts $(n=25)$ in the same cultures indicate that the majority of identified floating microtubules $(n=22$, from both 83 $\mathrm{nM}$ nocodazole overnight-treated and nocodazole-to- $8 \mathrm{nM}$ vinblastine experiments) and microtubules in axonal filopodia $(n=15)$ are single microtubules $(p>0.05$ for both floating microtubules and microtubules in axonal filopodia; Welch $t$ tests). The uniformity of the microtubule array of collateral branches was measured using what we term the width ratio (WR). Images of the microtubule array of collaterals were used to measure the width of the array at the base, within $5 \mu \mathrm{m}$ of the origin from the axonal microtubule array, and at its distal segment (usually within 5 $\mu \mathrm{m}$ of the distal-most extent of the array). In cases where the collateral's microtubule array was obviously thicker in the center than at either the base or the distal end, the width of the central region was substituted for that of the distal tip in the measurement of WR. This is reasonable because $\mathrm{WR}$ is intended as a measurement of the relative uniformity of the array. Formally, the ratio is expressed as WR = (width at the base of the collateral)/(width in the distal collateral).

Data presented in the text and figures are in the format of mean \pm SEM. All statistical analysis was performed using Instat software (Graphpad Software, San Diego, CA).

Gel electrophoresis and Western blotting for $\beta$-tubulin. Soluble and insoluble (polymerized) $\beta$-tubulin fractions were prepared according to established methods (Merrick et al., 1997). For each experiment, protein was collected from preplated neurons obtained from 100 ganglia. Briefly, neurons were scraped off the culturing substratum in $1 \mathrm{ml}$ of $40^{\circ} \mathrm{C}$ microtubule stabilization buffer (Merrick et al., 1997), and this material was then homogenized and centrifuged at $100,000 \times g$ for $20 \mathrm{~min}$ at $30^{\circ} \mathrm{C}$. This procedure generates a supernatant fraction containing soluble tubulin and a pellet containing polymerized tubulin. Protein samples were separated by SDS-PAGE (7.5\% gel) and electroblotted onto nitrocellulose membranes for Western blotting. $\beta$-tubulin was visualized using the same primary antibody described in Materials and Methods. Nitrocellulose membranes were first blocked for $1 \mathrm{hr}$ with Tris-buffered saline (TBS) containing 0.05\% Tween-20 (Sigma) (TBST) and 5\% nonfat dry milk (Nestle Food Company, Glendale, CA) (TBSTM) and then stained with a 1:100 dilution of the anti- $\beta$-tubulin antibody in TBSTM for $1 \mathrm{hr}$. The membranes were then washed once with TBST for $20 \mathrm{~min}$ and twice with TBST for $5 \mathrm{~min}$. Membranes were then stained with a goat antimouse HRP-conjugated secondary antibody (Jackson Immunoreseach Laboratories, West Grove, PA) at 1:500 in TBSTM for $1 \mathrm{hr}$, and again washed as described previously. HRP reaction products were obtained by incubating the membranes for $10 \mathrm{~min}$ with Supersignal Chemiluminescent kit reagents (Pierce, Rockford, IL), following the manufacturer's directions. The chemiluminescent signal was then detected by exposing the membranes to x-ray film (X-omat AR x-ray film, Eastman Kodak, Rochester, NY) for 1-10 sec, and the film was developed according to the manufacturer's directions. For presentation and analysis, the developed film was digitized using a flat-bed scanner (Hewlett Packard, Corvallis, OR). Densitometric analysis was performed using NIH Image 1.55. For the purpose of analysis, exposure times that did not saturate the signal in the darkest band on each gel were used.

\section{RESULTS}

\section{Dynamics of axonal sprouting and collateral formation}

Embryonic DRG axons in vitro spontaneously generate axon collateral sprouts (Gallo and Letourneau, 1998). We define axon collateral sprouts as protrusions that extend away from the axon. To better appreciate the dynamics of developing collateral sprouts we analyzed the growth pattern of sprouts using phasecontrast videomicroscopy. Based on our videomicroscopic observation of sprout formation, we adopted the following nomenclature to refer to different stages of axon collateral formation. Transient sprouts that did not extend beyond $10 \mu \mathrm{m}$ will be referred to as axonal filopodia (based on their similarity to growth cone filopodia). The term intermediate sprout will refer to sprouts that extended beyond the axonal filopodial stage but did not attain lengths $>30 \mu \mathrm{m}$ during the observation period. Sprouts that extended to $\geq 30 \mu \mathrm{m}$ will be referred to as collateral branches. The unqualified term collateral(s) will be used to refer to the general class of all axonal protrusions.

Collaterals arose as filopodia extended from the main axon (Fig. $1 A, B$ ), although only a small portion of axonal filopodia grew longer (Table 1), indicating a low probably for the transition from axonal filopodium to intermediate sprout or collateral branch stage. Furthermore, even collaterals that attained lengths $>15 \mu \mathrm{m}$ were not always retained. Observation of collaterals between 15 and $300 \mu \mathrm{m}$ long showed that collaterals $<40 \mu \mathrm{m}$ long were often quiescent, whereas longer collaterals exhibited steadier growth and less quiescence and retraction (Fig. $1 C$; Table 2 ) or remained quiescent for extended time periods (Fig. 1B). However, the rate of growth for collaterals that underwent extension during a $1 \mathrm{hr}$ period did not differ as a function of collateral length (Table 2). Periods of collateral elongation were associated with the dynamic extension of small lamellae or filopodia from the collateral tip. The necessity for actin-driven tip activity in extension and retraction of collaterals was verified by noting that $0.5 \mu \mathrm{g} / \mathrm{ml}$ cytochalasin $\mathrm{D}$, a treatment that inhibits actin filament polymerization, blocked both the extension and retraction of collateral branches ( $n=9$; data not shown). The extension of the main axons and collateral branches differed both in the rate of growth and the relative proportions of time spent actively growing, retracting, or remaining quiescent (Table 2). In contrast to axonal filopodia that had an average lifespan of $2.1 \pm$ $0.42 \min (n=87)$, no collaterals $>30 \mu \mathrm{m}$ were observed to fully retract during the $1 \mathrm{hr}$ observation period. Overall, our observations are consistent with previous descriptions of collateral branch formation in vitro (Sato et al., 1994; Yu et al., 1994; Szebenyi et al., 1998) and in vivo (Bastmeyer and O'Leary, 1996; Witte et al., 1996). In summary, the protrusion of an axonal filopodium rarely resulted in sustained growth of the axonal sprout. Initially, intermediate sprouts were relatively stable, whereas longer collateral branches exhibited more sustained growth.

\section{Cytoskeletal organization in collaterals}

Axonal filopodia exhibited F-actin staining similar to their counterparts at the growth cone (Fig. $2 A, B$ ). Intermediate sprouts and collateral branches often contained distal accumulations of F-actin (Fig. 2C), and our videomicroscopic observations suggest that variations in the actin content of the tips of collaterals reflect the activity of the collateral tip at the time of fixation. The shafts of collateral branches contained sparse F-actin staining, similar to the main axon.

We measured the frequency of microtubule invasion of collaterals as a function of collateral length. Only $15 \%$ of axonal filopodia contained microtubules (Fig. 2A,B; Table 3). The frequency of microtubule invasion of collaterals increased as a function of collateral length (Table 3 ). In almost all cases, microtubules extended to the tip of the sprout (Fig. $2 C$ ). In conjunction with our videomicroscopic observations, the data on microtubule localization to collaterals indicate that the stability and continued growth of collaterals may be related to the invasion of collaterals by microtubules. Short-lived axonal filopodia only rarely contain microtubules, whereas longer, more stable collaterals almost always contain microtubules.

The detyrosination of $\alpha$-tubulin is a time-dependent post- 

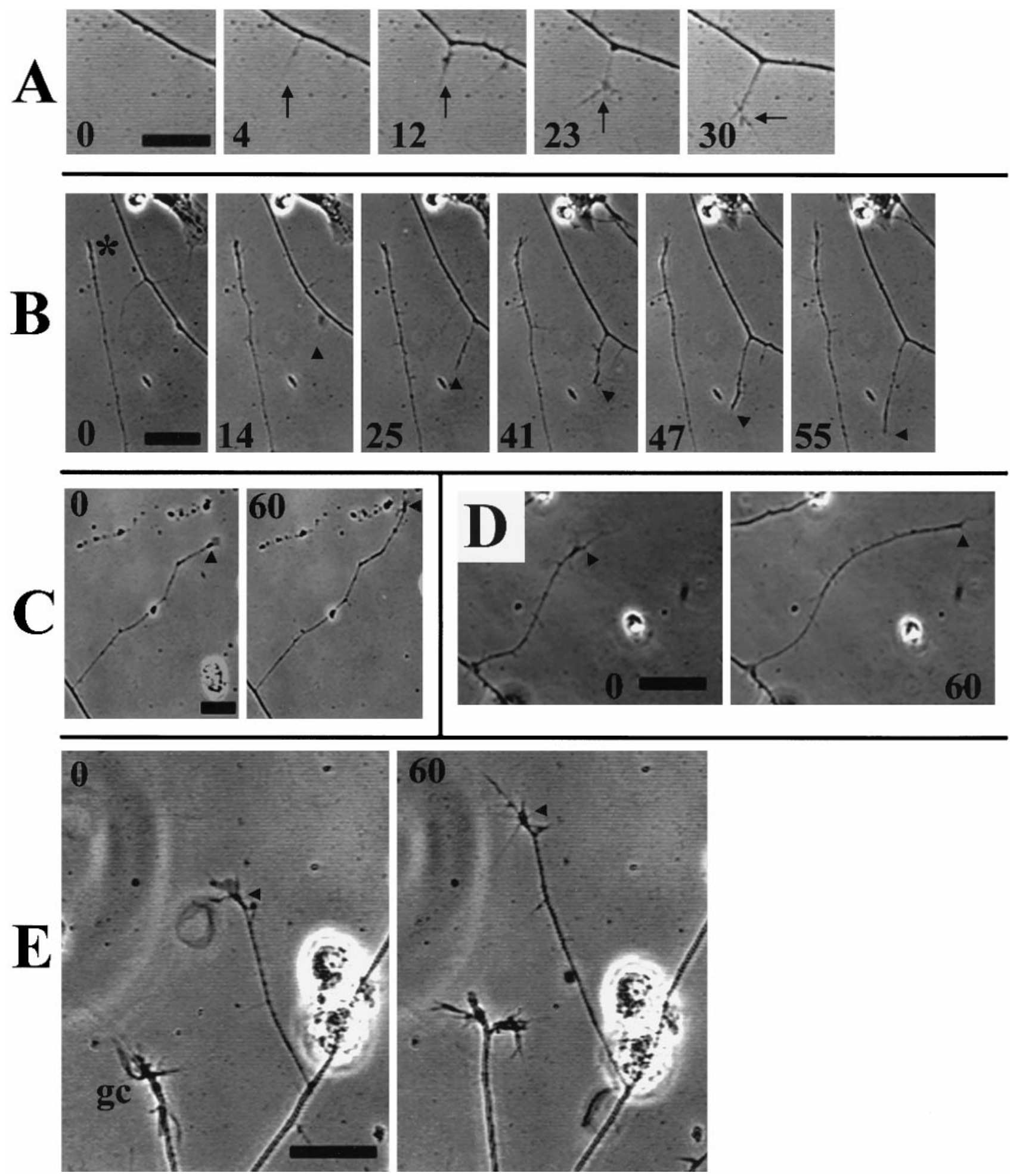

Figure 1. Dynamics of collateral extension by DRG axons. A, Collaterals were initiated from axons by the extension of axonal filopodia (minute 4 ), which subsequently thickened and developed small growth cone-like structures consisting mainly of short filopodial protrusions (minutes 23-30). Established collaterals were noted to remain quiescent for extended time periods $(B$, minutes $0-47 ; *$ denotes the tip of a collateral $75 \mu \mathrm{m}$ long at the beginning of the observation period) or to grow at a relatively steady rate $(C)$, over a $1 \mathrm{hr}$ observation period. Collaterals $>30 \mu \mathrm{m}$ in length often exhibited small lamellae, as well as filopodia, at their tips $(C, E)$. Similar to $A$, a collateral formed from the right-most axon in $B$ (minutes $14-55$, arrowheads). Collaterals of neurons raised in either $7 \mathrm{~nm}$ taxol $(D)$ or $83 \mathrm{~nm}$ nocodazole $(E)$ extended in a manner similar to those of controls. The arrowheads in $C-E$ denote the position of the collateral tip at the beginning of the observation period. Note the slow growth of the $83 \mathrm{~nm}$ nocodazole-treated axonal growth cone $(g c)$ in E. Numbers within individual panels reflect minutes after the initial panel, minute 0 , in the series. Scale bar, $10 \mu \mathrm{m}$. 


\begin{tabular}{lc}
\hline Table 1. Distribution of maximum attained axon collateral lengths \\
$\begin{array}{ll}\text { Collateral } & \% \text { of collaterals } \\
\text { length }(\mu \mathrm{m}) & 52 \\
\leq 5 & 35 \\
6-10 & 8 \\
11-15 & 3 \\
16-20 & 2 \\
>20 & \end{array}$
\end{tabular}

The behavior of individual filopodia that formed from an axonal shaft was monitored, and the maximal length the filopodium attained during the observation period was measured. A total of 199 axonal filopodia were observed from video records obtained from 14 separate axons from four separate cultures for a total of $2850 \mu \mathrm{m}$ of axon.

Table 2. Measurements of axon and collateral growth

\begin{tabular}{|c|c|c|c|c|}
\hline & \multicolumn{3}{|c|}{$\begin{array}{l}\text { Percentage of } 5 \mathrm{~min} \text { intervals } \\
\text { neurites engaged in }\end{array}$} & \multirow[b]{2}{*}{$\begin{array}{l}\text { Growth rate } \\
(\mu \mathrm{m} / \mathrm{hr})\end{array}$} \\
\hline & Growth & $\begin{array}{l}\text { Quie- } \\
\text { scence }\end{array}$ & Retraction & \\
\hline \multicolumn{5}{|l|}{ Control } \\
\hline Main axons (18) & 49 & 48 & 3 & $52 \pm 10(15)$ \\
\hline \multicolumn{5}{|l|}{ Collaterals } \\
\hline $15-40 \mu \mathrm{m}(29)$ & 8 & 87 & 5 & $10 \pm 2(10)$ \\
\hline$>40 \mu \mathrm{m}(15)$ & 25 & 66 & 9 & $16 \pm 5(8)$ \\
\hline \multicolumn{5}{|l|}{ Taxol (7 nm) } \\
\hline Main axons (16) & 29 & 66 & 5 & $27 \pm 6(12)^{a}$ \\
\hline \multicolumn{5}{|l|}{ Collaterals } \\
\hline$>40 \mu \mathrm{m}(16)$ & 20 & 73 & 7 & $21 \pm 4(8)$ \\
\hline \multicolumn{5}{|l|}{ NOC (83 nм) } \\
\hline Main axons (18) & 34 & 62 & 4 & $24 \pm 3(10)^{b}$ \\
\hline \multicolumn{5}{|l|}{ Collaterals } \\
\hline$>40 \mu \mathrm{m}(14)$ & 18 & 74 & 8 & $15 \pm 3(8)$ \\
\hline
\end{tabular}

Numbers in parentheses in the left-most column refer to the number of processes sampled. Because DMSO treatment was not found to alter any of the measurements, data were pooled and are referred to as control. The total number of $5 \mathrm{~min}$ intervals scored in each condition to obtain the percentage of time spent in growth, retraction, or quiescence ranged from 136 to 227 . Numbers in parentheses in the right-most column refer to the number of processes within each respective group that underwent growth during the observation period and were used to obtain mean growth rates. One to two axons in control, taxol-, and nocodazole-treated cultures, respectively, underwent continued retraction that was initiated soon after the start of the observation period and were omitted from the measurements. Growth rates of axon or collaterals raised in taxol or nocodazole were compared with control rates using the Welch $t$ test, one-tailed. No statistically significant differences were observed for growth rates of drug-treated collaterals relative to control collaterals, or between control collateral length classes. The $\chi^{2}$ analysis was used to compare the distributions of time collaterals spent growing, retracting, or being quiescent. Statistically significant differences were obtained when comparing control collaterals in the two length classes $(p<0.0001)$, and between the control and taxol- $(p<0.001)$ or nocodazole-treated axons $(p<0.01)$ but not taxol- or nocodazole-treated collaterals with length-matched controls.

${ }^{a} p<0.01$.

${ }^{b} p<0.05$.

translational modification of assembled tubulin that reflects the age, and in some cases the stability, of the microtubule polymer (Bulinski and Gundersen, 1991). As noted above, $15 \%$ of all axonal filopodia contained microtubules, and only $6 \%$ of axonal filopodia contained microtubules that were stained by detyrosinated $\alpha$-tubulin antibodies (hereafter referred to as $\mathrm{D} \alpha \mathrm{T}$ microtubules; Tables 3, 4). Longer collaterals contained D $\alpha \mathrm{T}$-labeled microtubules with increasing frequency, and microtubulecontaining collaterals $>20 \mu \mathrm{m}$ almost always contained D $\alpha \mathrm{T}$ labeled microtubules (Tables 3,4). Thus, the distribution of microtubules in collaterals supports the hypothesis that the relative instability of the early phases of collateral branch formation (i.e., axonal filopodia) reflects the lack of structural support provided by microtubules (Bastmeyer and O'Leary, 1996), whereas longer, more persistent collaterals are maintained by longer-lived $\mathrm{D} \alpha \mathrm{T}$-containing microtubules.

\section{Collateral formation is largely unaffected by concentrations of taxol and nocodazole that inhibit microtubule dynamics and axonal growth}

To determine the role of plus-end microtubule growth in the establishment of axon collaterals, we raised DRG neurons overnight (20 hr incubation) in drugs that attenuate plus-end microtubule growth and shrinkage in a dose-dependent manner (i.e., taxol and nocodazole) (Jordan and Wilson, 1998). Although the effects of raising DRG neurons in taxol have been reported previously (Letourneau et al., 1986), DRG neuronal growth in nanomolar concentrations of nocodazole, to our knowledge, has not been reported previously. Although 165-330 nм nocodazole reduced axonal length from embryonic rat superior cervical ganglion explants by $50 \%$ (Rochlin et al., 1996), we found that overnight growth of DRG neurons in $165 \mathrm{~nm}$ nocodazole resulted in no processes $>40 \mu \mathrm{m}$ ( $<10 \%$ of control values) and that axonal tips retracted when $165 \mathrm{~nm}$ nocodazole was acutely added to established cultures (data not shown). A 50\% reduction in axonal length occurred at 83 nм nocodazole, a concentration that attenuates microtubule dynamic instability (Vasquez et al., 1997). When DRG cultures were exposed overnight to vinblastine concentrations as low as $1 \mathrm{~nm}$, we consistently observed the death of all DRG cells, neuronal and non-neuronal (data not shown).

Both $7 \mathrm{~nm}$ taxol and $83 \mathrm{~nm}$ nocodazole increased the proportion of microtubules in collaterals that were double-labeled for total and $\mathrm{D} \alpha \mathrm{T}$ tubulin (Table 4), supporting previous conclusions that these drug concentrations reduce the presence of short-lived, dynamic microtubule polymer. Furthermore, the invasion of axonal filopodia by microtubules was reduced by $60 \%$ by both taxol and nocodazole (Table 3 ), indicating that microtubule plus-end polymerization promotes the invasion of microtubules into axonal filopodial sprouts. It is worth noting that both taxol and nocodazole decreased the frequency of microtubule invasion of axonal filopodia to the same 5-6\% level as the frequency of $\mathrm{D} \alpha \mathrm{T}$ microtubules in axonal filopodia under control conditions (Table 3 ), and the extent of $\mathrm{D} \alpha \mathrm{T}$ staining of microtubules found in axonal filopodia in drug-treated cultures is $144-240 \%$ of that in control cultures (Table 4). Thus, under normal conditions, longerlived $\mathrm{D} \alpha \mathrm{T}$-containing microtubules may enter axonal filopodia independently of microtubule plus-end dynamics.

The length of the main axonal shafts that formed after overnight culture was reduced to $40-50 \%$ of controls by either $7 \mathrm{nM}$ taxol or 83 nM nocodazole (Table 5). However, collateral branch length was much less reduced (Table 5), indicating a different role for microtubule plus-end dynamics in the growth of the main axon versus collateral branches. Furthermore, the growth rate and dynamics (time spent extending, retracting, or quiescent) of collateral branches of neurons raised in $7 \mathrm{~nm}$ taxol or $83 \mathrm{~nm}$ nocodazole were not different from control conditions (Fig. 1D,E; Table 2). Although the frequency of microtubule invasion of sprouts $<20 \mu \mathrm{m}$ long was decreased by taxol and nocodazole (Table 3), sprouts $>20 \mu \mathrm{m}$ in length contained microtubules with a frequency similar to control conditions (Table 3). Importantly, 

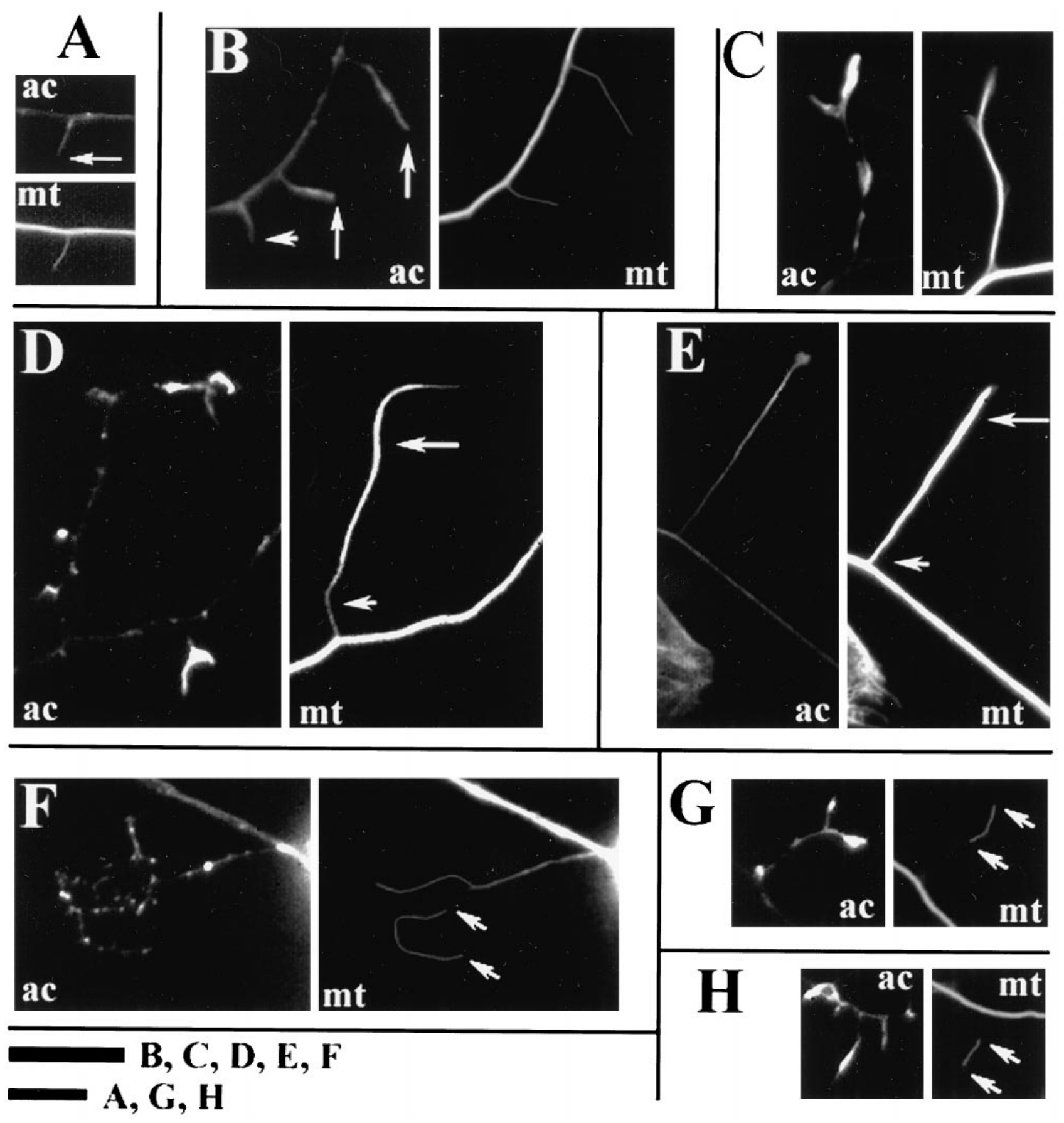

Figure 2. The cytoskeleton of collaterals. $A, B$, Axonal filopodia contained actin filaments, as determined by phalloidin staining (ac); $15 \%$ of axonal filopodia contained microtubules ( $m t$ ) (long arrows, $\beta$-tubulin staining). An example of an axonal filopodia not containing microtubules is denoted by the short arrow in $B$. Longer collaterals often exhibited actin filament accumulation at their tips, and the microtubule array was uniform $(C)$. The collaterals of neurons raised in $83 \mathrm{~nm}$ nocodazole $(D)$ or $7 \mathrm{~nm}$ taxol $(E)$ often exhibited distal accumulations of microtubules. Compare the staining of the microtubule array at the base of the collaterals (short arrow) with the distal segments (long arrows) in $D$ and $E$. These images were selected to show extreme cases of proximodistal disparity in the microtubule array. Furthermore, single microtubules separated from other microtubules (floating microtubules) were often observed in the collaterals of 83 nм nocodazole $(F)$ or 7 nm taxol $(G, H)$ raised neurons (arrows denote the tips of the floating microtubule). Scale bar, $10 \mu \mathrm{m}$. Letters next to bar in figure denote the panels to which the individual bar applies.

the frequency of formation of collaterals per $100 \mu \mathrm{m}$ of axon was not inhibited by either taxol or nocodazole (Table 6). The lack of an effect of $7 \mathrm{~nm}$ taxol on axonal collateral formation is particularly striking given that $7 \mathrm{~nm}$ taxol inhibits axonal branching that occurs by growth cone bifurcation (Letourneau et al., 1986; this study, data not shown). The frequency of short axonal protru- sions, as determined from phalloidin-stained neurons, was increased by both taxol and nocodazole (Table 6). However, videomicroscopic observations revealed that the majority of these "filopodia" were nonmotile membranous attachment points to the substratum and were not caused by increased filopodial formation rates along axons (11 and 13 axons sampled in DMSO and taxol, 
Table 3. Microtubule invasion of collaterals varies as a function of collateral length and is affected by raising neurons overnight in $7 \mathrm{nM}$ taxol or 83 nM nocodazole

\begin{tabular}{|c|c|c|c|c|}
\hline & \multicolumn{4}{|c|}{$\begin{array}{l}\% \text { of collaterals containing microtubules } \\
\text { (length in } \mu \mathrm{m} \text { ) }\end{array}$} \\
\hline & $<10$ & $11-20$ & $20-30$ & $>30$ \\
\hline \multicolumn{5}{|l|}{$\beta$-tubulin staining } \\
\hline No treatment & $15(236)$ & $54(111)$ & $76(26)$ & $93(69)$ \\
\hline DMSO & $14(886)$ & $54(183)$ & $79(56)$ & $99(96)$ \\
\hline Taxol (7 nм) & $6(1247)^{a}$ & $40(145)^{c}$ & $72(29)$ & $93(42)$ \\
\hline Nocodazole $(83 \mathrm{~nm})$ & $4(1186)^{a}$ & $36(285)^{b}$ & $76(71)$ & $94(84)$ \\
\hline \multicolumn{5}{|l|}{$\mathrm{D} \alpha \mathrm{T}$ staining } \\
\hline No treatment & $6(274)$ & $30(63)$ & $68(28)$ & $88(69)$ \\
\hline
\end{tabular}

Fisher's exact test was used to compare the frequency of microtubule invasion of collaterals in drug-treated cultures with DMSO controls. Numbers in parentheses reflect the number of collaterals in the given length class scored.

${ }^{a} p<0.0001$.

${ }^{b} p<0.001$.

${ }^{c} p<0.05$.

Table 4. Frequency of microtubules double-stained for $\beta$-tubulin and $\mathrm{D} \alpha$-T in collaterals

$\%$ of collateral microtubule(s) double-stained by $\beta$-tubulin and $\mathrm{D} \alpha \mathrm{T}$ antibodies (microtubule length in $\mu \mathrm{m}$ )

\begin{tabular}{lllrr} 
& $<10$ & $11-20$ & $20-30$ & $>30$ \\
\cline { 2 - 5 } Overnight & & & & \\
No treatment & $24(66)$ & $69(43)$ & $100(35)$ & $100(43)$ \\
DMSO & $27(115)$ & $57(35)$ & $96(23)$ & $100(21)$ \\
Taxol (7 nM) & $65(105)$ & $94(31)$ & $100(23)$ & $100(19)$ \\
$\quad$ Nocodazole (83 nM) & $39(119)$ & $83(98)$ & $94(36)$ & $100(26)$ \\
Acute treatment & & & & \\
NOC & $100(23)$ & $100(15)$ & $100(14)$ & $100(16)$ \\
NOC to & & & & \\
1 hr in 4 nM VB & $59(92)$ & $86(51)$ & $95(19)$ & $93(27)$ \\
1 hr in 8 nM VB & $67(45)$ & $90(31)$ & $100(15)$ & $97(30)$ \\
1 hr in 20 nM VB & $77(41)$ & $83(35)$ & $100(10)$ & $100(11)$ \\
\hline
\end{tabular}

NOC, $15 \mathrm{~min}$ in $2 \mathrm{mg} / \mathrm{ml}$ nocodazole; VB, vinblastine. Numbers in parentheses reflect the number of microtubule(s) in the given length class scored. Microtubules extending laterally from the main axonal array were scored in cultures doublestained with $\beta$-tubulin and $\mathrm{D} \alpha \mathrm{T}$ antibodies (see Materials and Methods). Microtubules were scored positive for $\mathrm{D} \alpha \mathrm{T}$ if they exhibited visible staining above the background staining observed in fibroblast lamellipodia within the same culture. The first four rows contain data collected from cultures treated with drugs overnight, whereas the remaining rows contain data from cultures acutely treated with drugs.

respectively; $p=0.97$, Welch $t$ test). Collectively, these data suggest that the formation and initial growth of sensory axon collaterals proceed in a relatively normal manner even when microtubule plus-end dynamics are much reduced.

In summary, treatment with low doses of taxol and nocodazole resulted in a $50 \%$ reduction in length of the main axon, but only slightly inhibited axon collateral growth. This is particularly significant because the drug treatments greatly inhibited microtubule localization to axonal filopodia and intermediate sprouts, the early phases of collateral branch formation. These observations demonstrate that the contribution of microtubule plus-end dynamics to collateral growth is relatively less than that to the growth of the main axon and indicate that the transport of microtubules may be significant in providing microtubules to the developing collateral sprouts.
Table 5. Effects of raising neurons in $7 \mathrm{nM}$ taxol or $83 \mathrm{~nm}$ nocodazole on axon and collateral lengths

\begin{tabular}{lll} 
Treatment & Axon length & Collateral length \\
\hline DMSO & $444 \pm 29(91)$ & $80 \pm 6(76)$ \\
Taxol & $259 \pm 23(68)^{a}$ & $69 \pm 5(65)^{b}$ \\
Nocodazole & $227 \pm 17(126)^{a}$ & $62 \pm 3(82)^{c}$
\end{tabular}

Numbers in parentheses reflect the number of axons or collaterals measured. Data were collected from six separate cultures. The Welch $t$ test was used to compare axon and collaterals lengths with DMSO values.

${ }^{a} p<0.0001$.

${ }^{b} p>0.05$.

${ }^{c} p<0.05$.

\section{Altered microtubule organization in collaterals of neurons raised in taxol or nocodazole}

The organization of microtubules in the collaterals of drugtreated axons was different from control cultures. Normally, collateral branches contained a microtubule array of uniform width and intensity along the branch (Fig. 2C). However, in the presence of $7 \mathrm{~nm}$ taxol or $83 \mathrm{~nm}$ nocodazole, microtubule mass often appeared greater distally than at the proximal base of collateral branches (Fig. 2D,E). This difference was confirmed quantitatively by dividing the width of the microtubule array at the collateral's base by the microtubule array width at the distal end of collaterals (WR = proximal/distal; see Materials and Methods). The WR of control (DMSO) collateral branches was $1.17 \pm$ $0.07(n=25)$ and for taxol-raised neurons (Welch $t$ test, onetailed, $p=0.0008)$ was $0.82 \pm 0.06(n=30)$. Furthermore, only $35 \%$ of control (DMSO) had WR measurements $<1.0$, whereas $80 \%$ of taxol-treated collateral branches had WR measurements $<1.0$. Conversely, $44 \%$ of control and $16 \%$ of taxol-treated collateral branches had WR measurements $>1.1$. The mean absolute width of the distal collateral microtubule array did not vary between control and taxol-treated collaterals (Welch $t$ test, twotailed, $p=0.14$ ), whereas the mean width of the microtubule array at the base of collaterals in taxol-treated neurons was significantly less than in controls (26\% difference; Welch $t$ test, two-tailed, $p=0.0085)$. These observations are consistent with a hypothesis that microtubule plus-end polymerization adds to the microtubule array of collateral branches, particularly at the proximal base of collaterals. However, the data also indicate that in the absence of normal microtubule plus-end growth, microtubules are still advanced in collaterals, probably by transport processes, until they reach the distal tip.

A striking feature of some axon collaterals of neurons grown overnight in taxol or nocodazole was the presence of individual microtubules with both ends visible and clearly separated from other microtubules. We will refer to these as floating microtubules (Fig. $2 F-H)$. When double-labeled for $\beta$-tubulin and $\mathrm{D} \alpha \mathrm{T}$, the majority of floating microtubules in $7 \mathrm{~nm}$ taxol $(83 \%, n=52)$ and $83 \mathrm{~nm}$ nocodazole $(76 \%, n=46)$ stained completely with $\mathrm{D} \alpha \mathrm{T}$ antibodies. Under control conditions, the frequency of collaterals containing such floating microtubules is significantly less (Table 7). Because these drug treatments alter the frequency with which collaterals contain microtubules, it is important to ask what percentage of collaterals containing microtubules exhibit floating microtubules. In both taxol and nocodazole this measurement was increased relative to controls (Table 7). The presence of floating microtubules in the collaterals of taxol- and nocodazoleraised neurons is consistent with the hypothesis that under conditions of reduced microtubule plus-end growth the elongation of 
Table 6. Frequency of collaterals formed along axons is not decreased by raising neurons in 7 nM taxol or 83 nм nocodazole

\begin{tabular}{lllll} 
& \multicolumn{4}{l}{ Collaterals per $100 \mu \mathrm{m}$ of axon (length in $\mu \mathrm{m})$} \\
\cline { 2 - 4 } Treatment & $<10$ & $11-20$ & $20-30$ & $>30$ \\
\hline DMSO (6) & $1.60 \pm 0.2$ & $0.36 \pm 0.1$ & $0.10 \pm 0.02$ & $0.17 \pm 0.03$ \\
Taxol (7 nM) (6) & $4.45 \pm 0.6^{a}$ & $0.53 \pm 0.1$ & $0.11 \pm 0.03$ & $0.13 \pm 0.03$ \\
Nocodazole (83 nM) (6) & $4.61 \pm 0.6^{a}$ & $1.19 \pm 0.2$ & $0.30 \pm 0.08$ & $0.33 \pm 0.10$ \\
\hline
\end{tabular}

Data were collected from six separate cultures from a total minimum of 100 neurons per treatment condition. Data was analyzed using an ANOVA with Bonferroni multiple comparison post hoc tests. Only axonal filopodia $(<10 \mu \mathrm{m}$ long $)$ were found to differ between taxol- or nocodazole-treated neurons and DMSO controls. Mean + SEM is shown.

${ }^{a} p<0.001$.

Table 7. Frequency of floating microtubule invasion of collaterals in the nocodazole-to-vinblastine drug regimen

\begin{tabular}{|c|c|c|c|}
\hline Treatment & $\begin{array}{l}\% \text { of collaterals } \\
\text { containing mts }\end{array}$ & $\begin{array}{l}\% \text { of collaterals } \\
\text { containing Fmts }\end{array}$ & $\begin{array}{l}\% \text { of collaterals } \\
\text { containing mts } \\
\text { with Fmts }\end{array}$ \\
\hline \multicolumn{4}{|l|}{ Overnight } \\
\hline Noc $(83 \mathrm{~nm})$ & $65(354)$ & $11(354)^{a}$ & $17(230)^{a}$ \\
\hline Tax (7 nM) & $67(380)$ & $13(380)^{a}$ & $19(255)^{a}$ \\
\hline DMSO & 79 (239) & $2(239)$ & $3(189)$ \\
\hline \multicolumn{4}{|l|}{ Acute treatment } \\
\hline \multicolumn{4}{|l|}{$\mathrm{NOC}$ to $4 \mathrm{VB}$} \\
\hline $1 \mathrm{hr}$ & $30(189)^{a}(42 \%)$ & $5(189)^{b}$ & $18(57)^{c}$ \\
\hline \multicolumn{4}{|l|}{ NOC to $8 \mathrm{VB}$} \\
\hline $15 \mathrm{~min}$ & $27(162)^{d}(54 \%)$ & $5(162)$ & $19(43)$ \\
\hline 1 hour & $41(86)^{a}(57 \%)$ & $10(86)^{d}$ & $25(36)^{a}$ \\
\hline \multicolumn{4}{|c|}{ NOC to $20 \mathrm{VB}$} \\
\hline $1 \mathrm{hr}$ & $16(128)^{a}(22 \%)$ & $5(128)$ & $33(21)^{d}$ \\
\hline \multicolumn{4}{|c|}{ NOC to DMSO } \\
\hline $15 \mathrm{~min}$ & $50(94)$ & $0(91)$ & $0(91)$ \\
\hline $1 \mathrm{hr}$ & $72(150)$ & $1(150)$ & $2(108)$ \\
\hline
\end{tabular}

NOC, Acute 15 min treatment with $2 \mu \mathrm{g} / \mathrm{ml}$ nocodazole; VB, vinblastine; Noc, nocodazole, overnight; Tax, taxol, overnight; mts, microtubule(s); Fmts, floating microtubule(s). For acute treatment experiments, data are presented for collaterals 11-30 $\mu \mathrm{m}$ long. For the overnight treatments with Noc, Tax, or DMSO, the data from all collaterals 11-200 $\mu \mathrm{m}$ long were pooled because no differences were found with regard to the frequency of Fmts in collaterals as a function of collateral length. The times under the treatment column denote the time exposed to VB or DMSO after the initial NOC treatment. Fischer's exact test was used to compare data within columns to the time-matched DMSO control. Because under control conditions no Fmts were observed $15 \mathrm{~min}$ after NOC, statistical comparisons with experimental measurements were not performed. Data were collected from a minimum of three separate experiments in each experimental condition. The percentages in parentheses (first data column) in the acute nocodazole-to-vinblastine treatments indicate the percentage of microtubule reinvasion of collaterals relative to the time-matched DMSO controls.

${ }^{a} p<0.0001$.

${ }^{b} p<0.05$.

${ }^{c} p<0.001$.

${ }^{d} p<0.01$.

collateral branches can be sustained by the continued distal transport of microtubules, which can become separated from each other by virtue of their shorter length caused by the inhibition of microtubule plus-end polymerization.

\section{Evidence for microtubule transport into collaterals when microtubule plus-end polymerization is inhibited}

To further investigate the transport of microtubules into axon collaterals, we used a drug regime to create conditions in which only stable microtubules are present and polymerization and plus-end dynamics are inhibited [see Ahmad and Baas (1995) and Ahmad et al. (1998) for a similar approach to the study of microtubule transport]. We treated cultures with $2 \mu \mathrm{g} / \mathrm{ml}$ nocodazole for periods ranging from 3 to $15 \mathrm{~min}$, a drug treatment previously used to depolymerize dynamic microtubule plus ends
(Table 8) (Baas and Heidemann, 1986; Baas and Black, 1990). Measurements of fractionated cytoskeletal preparations showed that by $15 \mathrm{~min}$ in $2 \mu \mathrm{g} / \mathrm{ml}$ nocodazole the amount of cytosolic unpolymerized $\beta$-tubulin was doubled (Fig. 3), consistent with the microtubule depolymerizing effects of nocodazole. In this state only $3 \%$ of intermediate sprouts contained microtubules, compared with 59\% under control conditions (Table 8; percentage was determined by considering all scored collaterals 11-30 $\mu \mathrm{m}$ long). Under these conditions all remaining microtubules in collaterals completely stained for $\mathrm{D} \alpha \mathrm{T}$ (Fig. 4A; Table 4) indicating that they consisted of older and relatively drug-stable polymer. When cultures were fixed immediately after the nocodazole treatment, floating microtubules were found in $\sim 20 \%$ of the microtubule-containing collaterals $>30 \mu \mathrm{m}$ long. All of the float- 


\begin{tabular}{|c|c|c|c|c|}
\hline \multirow{2}{*}{$\begin{array}{l}\text { Time after treat- } \\
\text { ment (min) }\end{array}$} & \multicolumn{4}{|c|}{$\begin{array}{l}\% \text { of collaterals containing microtubules } \\
\text { (length in } \mu \mathrm{m} \text { ) }\end{array}$} \\
\hline & $<10$ & $11-20$ & $20-30$ & $>30$ \\
\hline No treatment & $15(236)$ & $54(111)$ & $76(26)$ & $93(69)$ \\
\hline \multicolumn{5}{|l|}{ NOC only } \\
\hline 3 & $3(118)$ & $35(24)$ & $72(23)$ & $85(20)$ \\
\hline 6 & $1(98)$ & $34(29)$ & $60(15)$ & $78(14)$ \\
\hline 9 & $0(113)$ & $10(42)$ & $63(11)$ & $94(18)$ \\
\hline 15 & $0(147)$ & $2(54)$ & $6(16)$ & $57(19)$ \\
\hline \multicolumn{5}{|l|}{ NOC then $\mathrm{X}$} \\
\hline \multicolumn{5}{|l|}{ DMSO } \\
\hline 15 & $16(145)$ & $48(74)$ & $60(20)$ & ND \\
\hline 30 & $14(211)$ & $41(44)$ & $87(15)$ & ND \\
\hline 60 & $15(543)$ & $66(116)$ & $92(34)$ & ND \\
\hline \multicolumn{5}{|l|}{ Vinblastine (8 nM) } \\
\hline 15 & $3(248)$ & $19(101)$ & $39(61)$ & ND \\
\hline 60 & $8(210)$ & $40(65)$ & $48(21)$ & ND \\
\hline \multicolumn{5}{|c|}{ Vinblastine (20 nм) } \\
\hline 15 & $1(203)$ & $4(48)$ & $33(18)$ & ND \\
\hline 30 & $3(201)$ & $7(58)$ & $21(24)$ & ND \\
\hline 60 & $2(216)$ & $11(111)$ & $53(17)$ & ND \\
\hline
\end{tabular}

Cultures were treated with $2 \mu \mathrm{g} / \mathrm{ml}$ nocodazole (NOC). Data were collected from three separate experiments in each experimental condition. X, $15 \mathrm{~min}$ after NOC treatment; ND, not determined. As a baseline comparison point for the data on the effects of nocodazole and the time course of microtubule reinvasion, data from untreated cultures are also presented (same data as in Table 3).

ing microtubules we observed under these conditions $(n=34)$ were fully labeled by $\mathrm{D} \alpha \mathrm{T}$ antibodies.

Because continued exposure to $2 \mu \mathrm{g} / \mathrm{ml}$ nocodazole eventually results in the loss of all microtubule polymer, we removed nocodazole after an initial 15 min treatment and replaced it with 4-20 nM vinblastine to stabilize microtubules and inhibit repolymerization of microtubule plus ends (Jordan and Wilson, 1998). A $1 \mathrm{hr}$ treatment with 8 or $20 \mathrm{~nm}$ vinblastine alone was found to cause net microtubule depolymerization (Fig. 3), demonstrating that these concentrations of vinblastine inhibit microtubule dynamics. Furthermore, in response to $8-20 \mathrm{~nm}$ vinblastine, axonal growth cones either became quiescent or underwent a slight retraction (data not shown). After $1 \mathrm{hr}$ in 4-8 nM vinblastine, after the initial $15 \mathrm{~min}$ treatment with $2 \mu \mathrm{g} / \mathrm{ml}$ nocodazole, the majority of microtubules within collateral branches were fully stained for $\mathrm{D} \alpha \mathrm{T}$ (Table 4). Microtubules not staining for detyrosinated $\alpha$-tubulin were found mostly in sprouts $<10 \mu \mathrm{m}$ in length, consistent with the report that a small amount of polymerization occurs in the presence of 1-4 nM vinblastine (Miller and Joshi, 1996). A $1 \mathrm{hr}$ exposure to 8-20 nM vinblastine caused net microtubule depolymerization (Fig. 3) and resulted in the greatest coincidence of staining between $\beta$-tubulin and $\mathrm{D} \alpha \mathrm{T}$ along single microtubules (Table 4). Treatment for $1 \mathrm{hr}$ with $8 \mathrm{nM}$ vinblastine largely prevented microtubule reassembly after an initial $15 \mathrm{~min}$ treatment with $2 \mu \mathrm{g} / \mathrm{ml}$ nocodazole (Fig. 3; Table 8). Therefore, the regimen of a $15 \mathrm{~min}$ exposure to $2 \mu \mathrm{g} / \mathrm{ml}$ nocodazole followed by replacement of nocodazole with 4-20 nM vinblastine for $1 \mathrm{hr}$ resulted in neurons with largely nondynamic microtubules.

Then, to investigate the possibility of microtubule transport into axon collaterals, we determined whether collaterals that were made devoid of microtubules by the nocodazole treatment (i.e.,

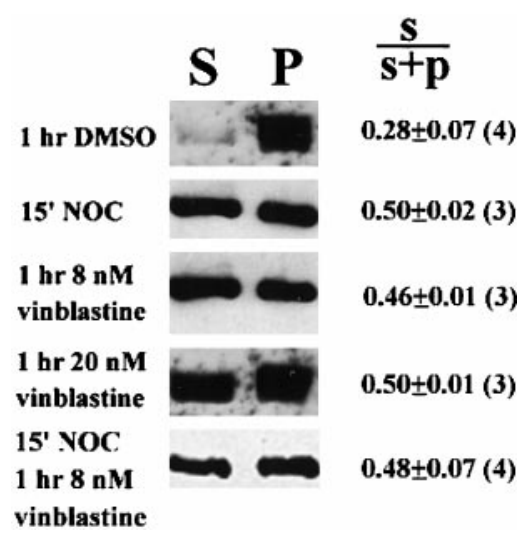

Figure 3. Effects of drug treatments on the relative amount of soluble/ cytoplasmic $(S)$ and polymerized $(P) \beta$-tubulin. The proportion of $S$ $\beta$-tubulin was quantified by obtaining the ratio of the total pixel intensity, over equal areas, of $S$ to $S+P$ (i.e., total) for each experiment. A $15 \mathrm{~min}$ treatment with $2 \mu \mathrm{g} / \mathrm{ml}$ nocodazole resulted in an increase in the proportion of $S \beta$-tubulin $(p<0.05)$ consistent with the microtubule depolymerizing action of nocodazole. A $1 \mathrm{hr}$ exposure to either 8 or $20 \mathrm{~nm}$ vinblastine also resulted in an increase in $S \beta$-tubulin ( $p<0.05$ for both), indicating that at these concentrations vinblastine caused net microtubule depolymerization. Treatment with $8 \mathrm{~nm}$ vinblastine for $1 \mathrm{hr}$, after an initial $15 \mathrm{~min}$ treatment with $2 \mu \mathrm{g} / \mathrm{ml}$ nocodazole, prevented the soluble tubulin fraction from returning to control levels. Equal amounts of sample buffer were loaded for $S$ and $P$ fractions within each experiment. The amount of protein loaded across experiments was not maintained constant, but this is not relevant because the measurement of interest is the relative proportion of $S$ tubulin within the experiment. $N O C=2 \mu \mathrm{g} / \mathrm{ml}$ nocodazole. The mean $\pm \operatorname{SEM}(n)$ of the $S / S+P$ ratios is shown in the figure. Welch $t$ tests were used to compare experimental values to the DMSO-treated controls.

11-30 $\mu \mathrm{m}$ long) were reinvaded by microtubules during treatment with 4-20 nu vinblastine, when axons contain stable microtubules and plus-end growth is inhibited. During a $1 \mathrm{hr}$ period in vinblastine after nocodazole treatments, axonal collaterals were reinvaded by microtubules (Tables 7, 8), but to a lesser degree than in cultures washed from nocodazole to DMSO (Tables 7, 8). Most neurons treated with 4-20 nm vinblastine appeared to show single microtubules extending into sprouts from the axonal array. Approximately $20 \%$ of microtubule-containing intermediate sprouts (11-30 $\mu \mathrm{m}$ long) contained floating microtubules (Table 7). As determined by double-labeling for $\mathrm{D} \alpha \mathrm{T}$ and $\beta$-tubulin, the majority of these floating microtubules completely stained for $\mathrm{D} \alpha \mathrm{T}$ (Fig. $4 B)(85 \%, n=40,94 \%, n=36$, and $88 \%, n=24$, for 4,8 , and $20 \mathrm{~nm}$ vinblastine, respectively). This reappearance of microtubules in intermediate sprouts that were made devoid of microtubules by nocodazole treatment is regarded by us as evidence for microtubule transport.

The hypothesis that microtubules are transported into collaterals in the nocodazole-to-vinblastine (4-20 nM) paradigm leads to several predictions. First, at shorter time intervals after release from nocodazole only the proximal regions of collaterals should contain microtubules. Second, with longer time periods in the presence of vinblastine, microtubules should accumulate in the distal portions of collaterals, as we observed for neurons raised overnight in taxol or nocodazole (see previous sections). Third, the first microtubules entering sprouts after release from nocodazole should stain completely for $\mathrm{D} \alpha \mathrm{T}$. Finally and importantly, the lengths of these microtubules should be greater than could be generated by the very low rate of polymerization that may occur in the presence of vinblastine. 

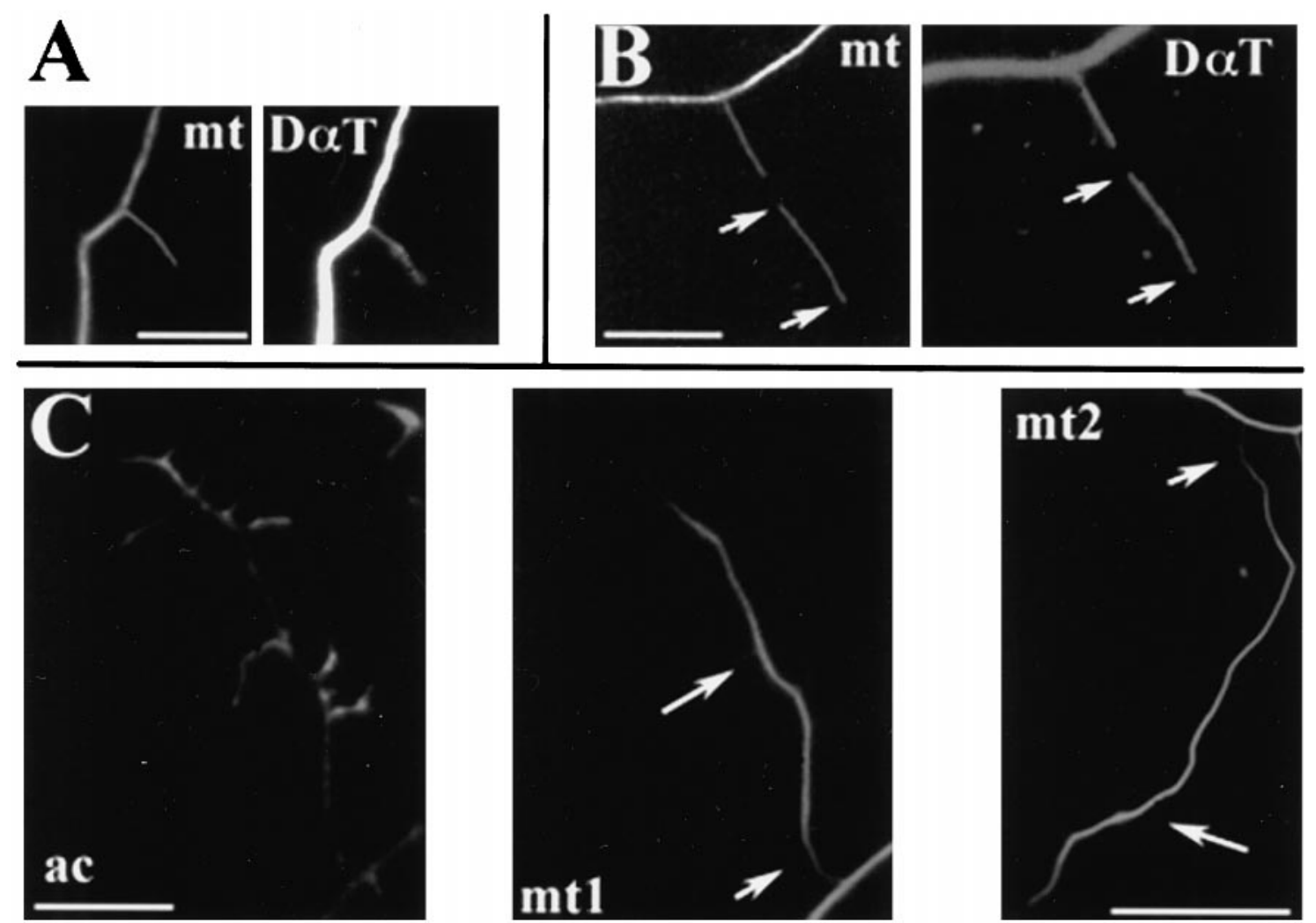

Figure 4. The microtubule cytoskeleton of collaterals in the nocodazole-to-vinblastine drug regimen. $A$, After a $15 \mathrm{~min}$ treatment with $2 \mu \mathrm{g} / \mathrm{ml}$ NOC, microtubules extending from the main axon stained fully with an antibody to detyrosinated $\alpha$-tubulin $(D \alpha T)(m t=\beta$-tubulin staining). $B$, Similarly, the majority of both the ends of microtubules extending from the main axon into collaterals and floating microtubules (arrows denote the tips of the floating microtubule), after a $1 \mathrm{hr}$ treatment with $8 \mathrm{~nm}$ vinblastine after release from NOC, also stained fully for D $\alpha \mathrm{T}$. $C$, Many collaterals of neurons treated for $2 \mathrm{hr}$ with $8 \mathrm{~nm}$ vinblastine after release from NOC exhibited distal accumulations of microtubules. $C$ ( $a c$ ) shows the actin cytoskeleton of such a collateral. Note the difference in microtubule content at the base (short arrow) and the distal segment of the collateral (long arrow) ( $C$, $m t 1)$. $C$, $m t 2$ shows another example of distal microtubule accumulation in a separate collateral. Scale bar, $10 \mu \mathrm{m}$.

All of these predictions were confirmed by measurements of microtubule invasion of collaterals after $15 \mathrm{~min}$ to $2 \mathrm{hr}$ in $8 \mathrm{nM}$ vinblastine after release from nocodazole. First, at 15 min after release from nocodazole in $61 \%$ of intermediate sprouts containing microtubules, the microtubules extended no more than $5 \mu \mathrm{m}$ from the axonal shaft into the collateral, whereas 60 min after release from nocodazole only $12 \%$ of collaterals with microtubules contained microtubules that were similarly confined to the proximal base of the collateral. Second, in cultures treated with 8 nM vinblastine for $2 \mathrm{hr}$ we observed an accumulation of microtubules in distal portions of collateral branches (Fig. 4C). Measurements of the width ratio (see previous sections and Materials and Methods) of the microtubule array of collaterals from cultures treated with $8 \mathrm{~nm}$ vinblastine for $2 \mathrm{hr}$ yield a mean WR of $0.72 \pm$ $0.04(n=24)$, similar to that observed in cultures raised overnight in taxol. Furthermore, after a $2 \mathrm{hr}$ treatment with $8 \mathrm{~nm}$ vinblastine, $16 \%$ of axons $(n=231)$ exhibited accumulations of microtubules in their distal portions that were separated from other microtubules by segments of axon devoid of microtubules (Fig. 5), whereas all the axons of neurons exposed to DMSO $(n=187)$ after the initial nocodazole treatment contained continuous microtubule arrays. This observation is further evidence for microtubule transport within the axon. Similar observations were made by Ahmad and Baas (1995) visualizing the axonal transport of microtubules. Third, the mean length of microtubules extending into the bases of collaterals from the main axonal array at $15 \mathrm{~min}$ after release from nocodazole in the presence of $8 \mathrm{~nm}$ vinblastine was $4.9 \pm 0.6 \mu \mathrm{m}(n=12$; range, $1.5-9.4 \mu \mathrm{m})$. This length is $181 \%$ of that expected by the calculations of Miller and Joshi (1996), who determined that in the presence of $4 \mathrm{~nm}$ vinblastine axonal microtubule polymerization occurs at $0.18 \pm 0.03 \mu \mathrm{m} / \mathrm{min}$ (mean $\pm \mathrm{SD}$ ), predicting a mean and maximum (4 SDs from the mean) lengths of 2.7 and $2.82 \mu \mathrm{m}$, respectively, of newly polymerized polymer during a 15 min period. Although Miller and Joshi (1996) did not report measurements of polymerization in the presence of $8-20 \mathrm{~nm}$ vinblastine, we expect that the polymerization rate may be even less under these conditions.

Furthermore, the majority of microtubules that reinvaded collaterals in the presence of vinblastine after release from nocodazole contained $\mathrm{D} \alpha \mathrm{T}$ and exhibited lengths too great to be accounted for by the reduced rate of polymerization. After $15 \mathrm{~min}$ in $8 \mathrm{~nm}$ vinblastine, $77 \%$ of microtubules $(n=17)$ extending into collaterals from the main axon array were completely doublestained for $\beta$-tubulin and D $\alpha \mathrm{T}$. Because the detyrosination of $\alpha$-tubulin after incorporation into polymer requires 20-30 min (Gundersen et al., 1987), it is highly unlikely that within $15 \mathrm{~min}$ after removing nocodazole these microtubules were first polymerized at a slow rate and then detyrosinated. The mean length of microtubule ends without detectable staining for $\mathrm{D} \alpha \mathrm{T}$ that extended from the main axonal array into the bases of collaterals was $2.0 \pm 0.5 \mu \mathrm{m}(n=5)$, consistent with the low polymerization rate in the presence of vinblastine reported by Miller and Joshi 
Figure 5. Evidence for microtubule transport in axons. After a $2 \mathrm{hr}$ treatment with $8 \mathrm{~nm}$ vinblastine after release from a $15 \mathrm{~min}$ treatment with $2 \mu \mathrm{g} / \mathrm{ml}$ nocodazole, some axons exhibited heterogeneities of the microtubule array suggesting the centrifugal transport of microtubules. $A$ shows a neuron $(\mathrm{sm}=$ soma) with a distinct microtubule accumulation in one of its axonal branches (ax1). Note that there is an apparent lack of microtubules in the proximal portion of the axon (arrow), whereas the distal portion contains a microtubule array (large arrow). A small branch originating from the distal portion of ax contains a floating microtubule (arrowhead) (because of the length of the branch and proximity to the axonal tip, this branch is not considered to be a collateral; see Materials and Methods). ax2 also exhibits a heterogeneity in its microtubule array. The arrow indicates a region of the proximal axon relatively poor in microtubule polymer. ax3 did not exhibit any obvious heterogeneities in its microtubule array. Two collateral branches originating from $a \times 2$ (cll and $c l 2$ ) also do not show a distal accumulation of microtubules. $B$ shows another example of an axon (axl) with a distal accumulation of microtubules (large arrow) separated from the rest of the mi-
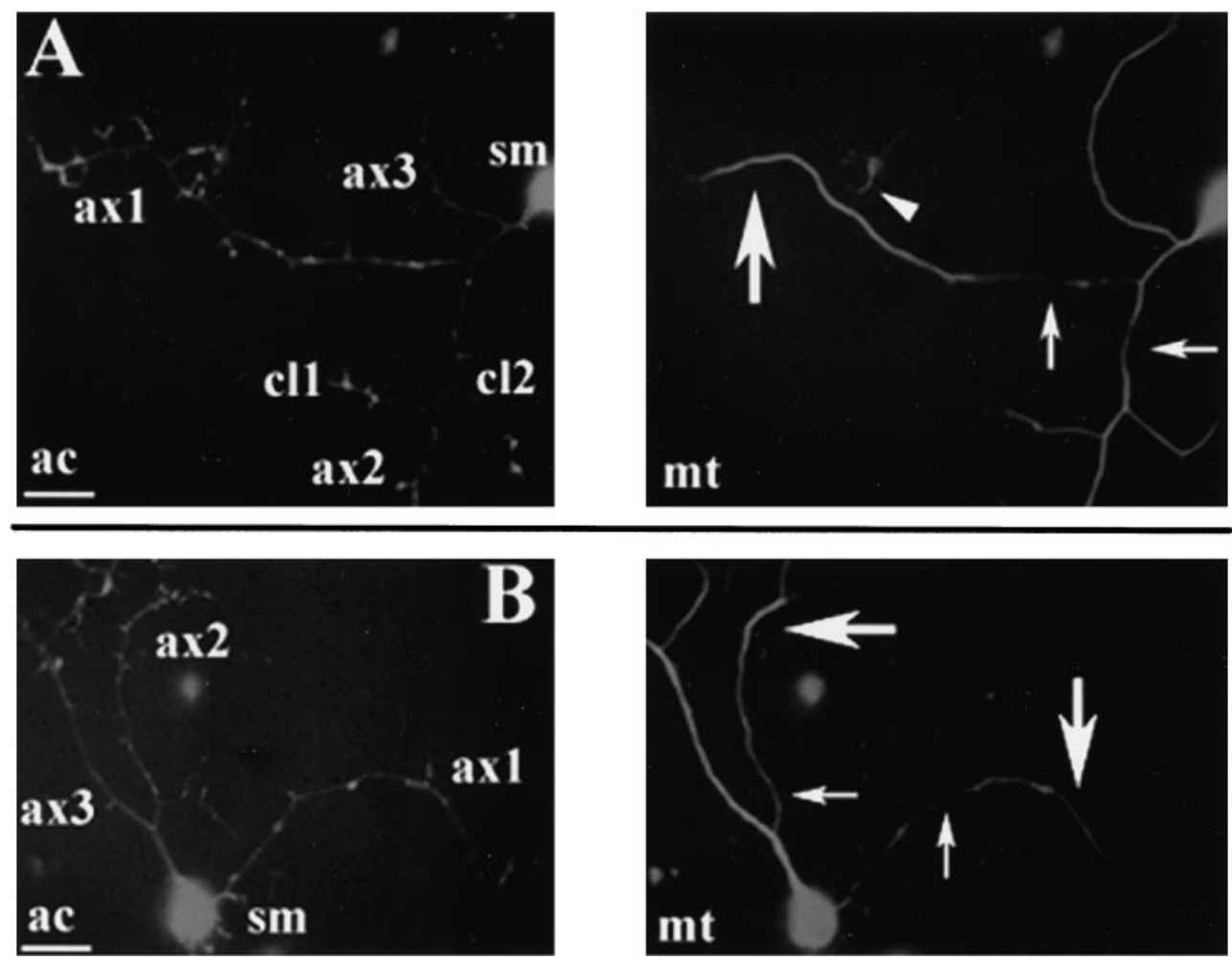

crotubule array by a region of axon devoid of microtubules (small arrow). ax2 in $B$ shows an example of an axon with a distal accumulation of microtubules (compare the thickness of the array at the large and small arrow), but the array remains continuous throughout the axon. The microtubule array of ax 3 in $B$ does not show any evident heterogeneity. Note that the actin staining does not reveal any obvious damage to the regions of axons that are relatively poor in microtubules. Scale bar, $10 \mu \mathrm{m}$.

(1996). The mean length of such microtubule ends staining for $\mathrm{D} \alpha \mathrm{T}$ (Fig. $4 B$ ) was significantly greater than that of microtubule ends lacking $\mathrm{D} \alpha \mathrm{T}$ staining (Welch $t$ test, two-tailed, $p=0.002$ ). Similar results were obtained for the floating microtubules present at $15 \mathrm{~min}$ after release from nocodazole in the presence of $8 \mathrm{~nm}$ vinblastine. Six of seven floating microtubules, present 15 min after release from nocodazole and in the continuous presence of $8 \mathrm{~nm}$ vinblastine, stained completely for $\mathrm{D} \alpha \mathrm{T}$ and had a mean length of $6.3 \pm 1.5 \mu \mathrm{m}$ (range, $4.5-13 \mu \mathrm{m}$ ), whereas the one floating microtubule without clear $\mathrm{D} \alpha \mathrm{T}$ staining was only $1.6 \mu \mathrm{m}$ long. We performed similar measurements on microtubules within collaterals in experiments in which cultures were treated first with nocodazole and then with $20 \mathrm{~nm}$ vinblastine for $15 \mathrm{~min}$ and obtained similar results. Under these conditions, D $\alpha \mathrm{T}$ containing microtubules that extended into the bases of collaterals had a mean length of $9.1+0.8 \mu \mathrm{m}(n=26)$, and D $\alpha \mathrm{T}$ containing floating microtubules had lengths of $9.2+1.7 \mu \mathrm{m}(n=$ 7). The ends of microtubules extending from the main axonal array into the bases of collaterals that did not stain for $\mathrm{D} \alpha \mathrm{T}$ had a mean length of only $2.92+0.4 \mu \mathrm{m}(n=6)$. Under these conditions we did not observe floating microtubules that did not contain $\mathrm{D} \alpha \mathrm{T}$. In another set of experiments, cultures were treated first with nocodazole, then washed and treated for 15 min with 20 nM vinblastine, fixed, and then double-stained with phalloidin and $\mathrm{D} \alpha \mathrm{T}$-antibodies. This allowed us to determine the lengths of $\mathrm{D} \alpha \mathrm{T}$-stained microtubules contained in intermediate sprouts (11-30 $\mu \mathrm{m}$ long). Microtubules extending from the main axonal array into intermediate sprouts had a mean length of $8.9 \pm 0.9$ $\mu \mathrm{m}(n=22)$, and floating microtubules had a mean length of $9.8 \pm 1.5 \mu \mathrm{m}(n=9)$, consistent with our previous measurements of microtubule lengths in cultures double-stained for $\beta$-tubulin and $\mathrm{D} \alpha \mathrm{T}$.

Therefore, these measurements indicate that the contribution of microtubule plus-end polymerization to the localization of microtubules in collaterals in the nocodazole-to-vinblastinetreated neurons was too little to account for the observed timedependent microtubule reinvasion of collaterals. Collectively, these data indicate that in the presence of vinblastine, after nocodazole treatment, nondynamic microtubules underwent transport from the axon into collaterals.

Although we favor the transport of microtubules as the mechanism for the reappearance of microtubules in collaterals in the nocodazole-to-vinblastine experiments, alternatives must be considered. Given that vinblastine largely inhibited microtubule plusend growth in these experiments, it seems unlikely that the microtubules could have treadmilled into the collaterals (Jordan and Wilson, 1998). Similarly, it is unlikely that microtubules polymerized de novo within the collaterals, because during $1 \mathrm{hr}$ in $4 \mathrm{nM}$ vinblastine the maximum expected length of new microtubule polymer is $11.8 \mu \mathrm{m}$ (as determined from the calculations detailed in the previous paragraph), and the relative percentage of collaterals $>30 \mu \mathrm{m}$ in length that contained floating microtubules $<12 \mu \mathrm{m}$ long did not change during $1 \mathrm{hr}$ of $4 \mathrm{~nm}$ vinblastine treatment (data not shown). Consistent with this analysis, Baas and Heidemann (1986) investigated microtubule nucleation and reassembly after complete depolymerization induced by $15 \mathrm{~min}$ in $1 \mu \mathrm{g} / \mathrm{ml}$ nocodazole and found no evidence of microtubule self-assembly even $30 \mathrm{~min}$ after release from nocodazole. The most reasonable and parsimonious explanation for how floating microtubules came to reside within sprouts is that they were 
transported there in the absence of significant microtubule growth by plus-end polymerization.

\section{DISCUSSION}

Axon collateral branch formation is an important mechanism by which neuronal connectivity patterns are established. However, the cytoskeletal mechanisms underlying collateral branch formation are poorly understood [see Yu et al. (1994) for an outstanding exception]. In this report we demonstrate that (1) both microtubule dynamics and transport contribute to the formation of the microtubule array of sensory axon collaterals, and (2) the relative importance of these activities (microtubule transport and polymerization) differs between collateral branches and the main axon. We also provide experimental evidence for the transport of microtubules from axons into collaterals.

The existence of microtubule transport in neurons, and its role in axonal growth, has been the focus of much recent debate [see Baas (1997) and Joshi (1998) for opposing arguments]. Our data are consistent with the occurrence of microtubule transport in axons and to our knowledge are the first experimental demonstration of transport into collaterals [however, see Yu et al. (1994) for a correlative study of microtubule age and collateral invasion]. Although we find differences in the relative extent to which microtubule polymerization and transport contribute, respectively, to neurite growth of axons versus collateral branches, we emphasize that our data indicate that both transport and dynamic instability contribute to the microtubule array of collateral branches. We suggest that the "normal" growth of axons [as argued by Black (1994) and Baas (1997)] and collateral branches uses both microtubule dynamics and transport to establish the microtubule array, but that the two appear to have different roles in the growth of axons and collateral branches.

The growth of axon collaterals was less sensitive to treatments that inhibit microtubule plus-end dynamics than the growth of the main axon. These data indicate that the growth mechanisms of the main axon differ from those of the collateral branch. Typically, the growth cone of the main axon remained active for extended periods of axonal elongation, whereas the tips of collaterals exhibited more sporadic periods of activity interspersed with inactivity. However, as demonstrated by experiments using cytochalasin $\mathrm{D}$, the growth of both collaterals and the main axon depends on this actin-based motility. Importantly, although overnight treatment with $7 \mathrm{~nm}$ taxol or $83 \mathrm{~nm}$ nocodazole inhibited microtubule entry into axonal filopodia by $60 \%$, axonal collateral branches formed and extended in a relatively normal manner. These data strongly argue that the entry of microtubules into collaterals by plus-end polymerization and dynamics during the early phases of collateral branch formation makes a minor contribution to the process by which collateral branches are established. In a similar conclusion, Smith (1994) reported that the initiation of neurites from the soma of sympathetic neurons is independent of microtubule dynamics and can rely on the translocation of stable polymer from the soma into the process. Interestingly, neurites were initiated from the soma as long filopodia (Smith, 1994), also similar to the formation of collateral branches from an axon. Therefore, both axons and collaterals may be initiated through mechanisms independent of microtubule plusend assembly. However, after the initial stages, the normal continued growth of axons, but not collaterals, depends to a greater extend on microtubule plus-end assembly.

By using nocodazole to eliminate microtubules from collaterals $<30 \mu \mathrm{m}$ long and then by inhibiting plus-end microtubule growth with vinblastine, we were able to experimentally demonstrate that axonal microtubules are transported into axon collateral branches. The evidence of Yu et al. (1994) previously suggested microtubule transport into axon collateral branches because the age of the microtubule polymer in nascent collaterals did not differ from that in the main axon. However, differences appear between the rat hippocampal neurons studied by Yu et al. (1994) and chick embryonic DRG neurons. Yu et al. (1994) report that almost all hippocampal axonal sprouts 5-25 $\mu \mathrm{m}$ long contained microtubules that were at least as old as those present in the axon. However, only $\sim 40 \%$ of DRG axon collateral sprouts $<20 \mu \mathrm{m}$ long contained long-lived microtubules, as determined by $\mathrm{D} \alpha \mathrm{T}$ staining. Furthermore, our evidence that inhibition of microtubule plus-end dynamics by both taxol and nocodazole decreased the frequency of microtubule invasion of DRG axonal filopodia by $60 \%$ suggests that dynamic microtubule plus ends provide a large contribution to the entry of microtubules into nascent DRG axon collaterals, relative to hippocampal neurons. Yet, consistent with the observations of Yu et al. (1994), DRG axonal sprouts $>20 \mu \mathrm{m}$ long consistently contained D $\alpha \mathrm{T}$-immunoreactive microtubules. The disparity between our data and that of $\mathrm{Yu}$ et al. (1994) may reside in differences in neuronal type or culturing conditions (e.g., substratum type). Consistent with the latter suggestion, a recent report by Chang et al. (1998) indicates that the modulation of neurite tension by adhesion to a substratum may significantly alter the rate of axonal microtubule turnover and transport.

Our data are consistent with the hypothesis of the transport of axonal microtubules into collaterals (Yu et al., 1994) and provide further support for the hypothesis of microtubule transport in neuronal processes (Baas, 1997). The presence of floating microtubules in the collaterals of axons raised in $7 \mathrm{nM}$ taxol or $83 \mathrm{nM}$ nocodazole provides compelling evidence in favor of the transport of microtubules. Furthermore, the presence of increased microtubule mass in the distal portions of collateral branches raised in $7 \mathrm{~nm}$ taxol or $83 \mathrm{~nm}$ nocodazole, or after prolonged periods in vinblastine after release from nocodazole, also lends itself to the interpretation that under these conditions microtubules are transported and accumulate within collaterals. In our experiments, the strongest evidence for microtubule transport comes from the experimental paradigm of evacuating microtubules from collaterals with $2 \mu \mathrm{g} / \mathrm{ml}$ nocodazole followed by microtubule reinvasion of collaterals in the presence of 4-20 nM vinblastine.

Collectively, the data presented here suggest a working model of the cytoskeletal events underlying the formation of axon collaterals from DRG axons. (1) An initial reorganization of the axonal cortical actin cytoskeleton results in the formation of transient axonal filopodia. (2) Dynamic microtubules invade transient axonal filopodia. (3) The ends of axonal microtubules undergoing transport may also invade axonal filopodia. Unless dynamic microtubules become stabilized while within an axonal filopodium, by their very nature they are likely to depolymerize and vacate the axonal filopodium, leaving it without internal support. Furthermore, dynamic microtubule ends may not be able to support or counteract actin-mediated forces produced when an axonal filopodium retracts. However, when stable microtubules are transported into axonal filopodia, or dynamic microtubules are stabilized within the filopodia, a stronger structural support is provided for the actin cytoskeleton of the nascent collateral. We suggest that the stability of microtubules in nascent collaterals is fundamental for the subsequent growth of the collateral. Consis- 
tent with this hypothesis, overnight culturing in taxol or nocodazole at concentrations that attenuate microtubule plus-end dynamics did not alter the frequency at which axon collaterals formed and had only minor effects on the lengths they were able to attain, demonstrating that normal microtubule dynamics within the axon are not required for collateral branch formation. The finding that inhibition of microtubule plus-end dynamics by $7 \mathrm{~nm}$ taxol and 83 nm nocodazole greatly reduced the overall percentage of axonal filopodia that contained microtubules (Table 3 ) but did not affect the frequency of $\mathrm{D} \alpha \mathrm{T}$-containing microtubules in axonal filopodia (Table 4) suggests that the localization of $\mathrm{D} \alpha \mathrm{T}$ microtubules into axonal filopodia, perhaps by transport mechanisms, may be a key event in the early stages of collateral growth. (4) Once an axonal filopodium becomes stabilized by one or more stable microtubules, microtubule plus-end dynamics then contribute mass to the microtubule array of the growing collateral. Assuming that stable microtubules provide structural support for the collateral's actin cytoskeleton, this may provide an intracellular environment that favors further microtubule addition to the nascent branch by decreasing the likelihood that retraction of the filopodial actin cytoskeleton and the associated forces may drive microtubule depolymerization (Heidemann and Buxbaum, 1994; Kaech et al., 1996). Indeed, removal of actin filaments from growth cones allows microtubules to extend deeper into the periphery (Forscher and Smith, 1988), indicating that aspects of the actin cytoskeleton may be able to negatively regulate the ability of dynamic microtubules to invade cellular domains. (5) Microtubules are then continuously transported into developing collaterals and become more stable, providing further support structures for the collateral. This model makes two fundamental predictions: (1) conditions that increase the numbers of transported microtubules should increase the likelihood of microtubule transport into transient axonal sprouts and thereby increase the probability of any given axonal filopodium to become stabilized and undergo subsequent growth. (2) Conversely, conditions that decrease the transport of stable microtubule polymer or prevent microtubule stabilization should decrease the frequency of collateral branch formation.

The relative independence of axon collateral growth from microtubule dynamic instability has important implications for neurodevelopment. Axon collaterals are usually formed from regions of the axon up to millimeters behind the growth cone (O'Leary and Koester, 1993). The dynamic instability of axonal microtubules decreases during axonal maturation (Lim et al., 1989; Baas et al., 1993; Edson et al., 1993), indicating that axon collaterals in vivo form from regions of the axon that contain mostly stable tubulin polymer. The independence of collateral branch formation from dynamic microtubule growth would allow collateral branch formation without the need to locally increase microtubule dynamic instability. The relative lack of dependence of axon collateral formation on microtubule dynamic instability may therefore be an efficient mechanism to maintain the ability of neurons to establish connections with their targets.

\section{REFERENCES}

Ahmad FJ, Baas PW (1995) Microtubules released from the neuronal centrosome are transported into the axon. J Cell Sci 108:2761-2769.

Ahmad FJ, Echeverri CJ, Vallee RB, Baas PW (1998) Cytoplasmic dynein and dynactin are required for the transport of microtubules into the axon. J Cell Biol 140:391-401.

Baas PW (1997) Microtubules and axonal growth. Curr Opin Cell Biol 9:29-36.
Baas PW, Ahmad FJ (1993) The transport properties of axonal microtubules establish their polarity orientation. J Cell Biol 120:1427-1437.

Baas PW, Black MM (1990) Individual microtubules in the axon consist of domains that differ in both composition and stability. J Cell Biol 111:495-509.

Baas PW, Heidemann SR (1986) Microtubule reassembly from nucleating fragments during the regrowth of amputated neurites. J Cell Biol 103:917-927.

Baas PW, Ahmad FJ, Pienkowski TP, Brown A, Black MM (1993) Sites of microtubule stabilization for the axon. J Neurosci 13:2177-2185.

Bastmeyer M, O'Leary DM (1996) Dynamics of target recognition by interstitial axon branching along developing cortical axons. J Neurosci 16:1450-1459.

Bhide PG, Frost DO (1991) Stages of growth of hamster retinofugal axons: implications for developing axonal pathways with multiple targets. J Neurosci 11:485-504.

Black MM (1994) Microtubule transport and assembly cooperate to generate the microtubule array of growing axons. Prog Brain Res 102: 61-77.

Bulinski JC, Gundersen GG (1991) Stabilization and post-translational modification of microtubules during cellular morphogenesis. BioEssays 13:285-293.

Castellani V, Yue Y, Gao P, Zhou R, Bolz J (1998) Dual action of a ligand for Eph receptor tyrosine kinases on specific populations of axons during the development of cortical circuits. J Neurosci 18:4663-4672.

Challacombe JF, Snow DM, Letourneau PC (1997) Dynamic microtubule ends are required for growth cone turning to avoid an inhibitory guidance cue. J Neurosci 17:3085-3110.

Chang S, Rodionov VI, Borisy GG, Popov SV (1998) Transport and turnover of microtubules in frog neurons depend on the pattern of axonal growth. J Neurosci 18:821-829.

Edson KJ, Lim S, Borisy GG, Letourneau PC (1993) FRAP analysis of the stability of the microtubule population along the neurites of chick sensory neurons. Cell Motil Cytoskeleton 25:59-72.

Forscher P, Smith SJ (1988) Actions of cytochalasins on the organization of actin filaments and microtubules in a neuronal growth cone. J Cell Biol 107:1505-1516.

Gallo G (1998) Involvement of microtubules in the regulation of growth cone morphologic remodeling. J Neurobiol 35:121-140.

Gallo G, Letourneau PC (1998) Localized sources of neurotrophins initiate axon collateral branching. J Neurosci 18:5403-5414.

Gallo G, Lefcort FB, Letourneau PC (1997) The trkA receptor mediates growth cone turning toward a localized source of nerve growth factor. J Neurosci 17:5445-5454.

Ghosh A, Shatz CJ (1992) Pathfinding and target selection by developing geniculocortical axons. J Neurosci 12:39-55.

Gundersen GG, Khawaja S, Bulinski JC (1987) Postpolymerization detyrosination of alpha-tubulin: a mechanism for subcellular differentiation of microtubules. J Cell Biol 105:251-264.

Heffner CD, Lumsden AGS, O'Leary DDM (1990) Target control of collateral extension and directional axon growth in the mammalian brain. Science 247:217-220.

Heidemann SR, Buxbaum RE (1994) Mechanical tension as a regulator of axonal development. Neurotoxicology 15:95-108.

Jordan MA, Wilson L (1998) Microtubules and actin filaments: dynamic targets for cancer chemotherapy. Curr Opin Cell Biol 10:123-130.

Joshi HC (1998) Microtubule dynamics in living cells. Curr Opin Cell Biol 10:35-44.

Kadhim H, Bhide P, Frost DO (1993) Transient axonal branching in the developing corpus callosum. Cereb Cortex 3:551-556.

Kaech S, Ludin B, Matus A (1996) Cytoskeletal plasticity in cells expressing neuronal microtubule-associated proteins. Neuron 17:1189-1199.

Kennedy TE, Tessier-Lavigne M (1995) Guidance and induction of branch formation in developing axons by target-derived diff usible factors. Curr Opin Neurobiol 5:83-90.

Letourneau PC, Shattuck TA, Ressler AH (1986) Branching of sensory and sympathetic neurites in vitro is inhibited by treatment with taxol. J Neurosci 6:1912-1917.

Lim S, Sammak PJ, Borisy GG (1989) Progressive and spatially differentiated stability of microtubules in developing neuronal cells. J Cell Biol 109:253-263.

Merrick SE, Trojanowski JQ, Lee VM-Y (1997) Selective destruction of stable microtubules and axons by inhibitors of protein serine/threonine 
phosphatases in cultures human neurons (NT2N cells). J Neurosci 17:5726-5737.

Miller KW, Joshi HC (1996) Tubulin transport in neurons. J Cell Biol 133:1355-1366.

Okabe S, Hirokawa N (1992) Differential behavior of photoactivated microtubules in growing axons of mouse and frog neurons. J Cell Biol 117:105-120.

O'Leary DDM, Koester SE (1993) Development of projection neuron types, axon pathways, and patterned connections of the mammalian cortex. Neuron 10:991-1006.

O'Leary DDM, Terashima T (1988) Cortical axons branch to multiple targets by interstitial axon budding: implications of target recognition and "waiting periods". Neuron 1:901-910.

O'Leary DDM, Heffner CD, Kutka L, Lopez-Mascaraque L, Missias A, Reinsoso BS (1991) A target derived chemoattractant controls the development of the corticopontine projection by a novel mechanism of axon targeting. Development [Suppl] 2:123-130.

Ozaki S, Snider WD (1997) Initial trajectories of sensory axons toward laminar targets in the developing mouse spinal cord. J Comp Neurol 380:215-229.

Reinsch SS, Mitchison TJ, Kirschner M (1991) Microtubule polymer assembly and transport during axonal elongation. J Cell Biol 115:365-379.

Rochlin MW, Wickline KM, Bridgman PC (1996) Microtubule stability decreases axon elongation but not axoplasm production. J Neurosci 16:3236-3246.

Sato M, Lopez-Mascaraque L, Heffner CD, O’Leary DM (1994) Action of a diffusible target-derived chemoattractant on cortical axon branch induction and directed growth. Neuron 13:791-803.

Schwegler G, Schwab ME, Kapfhammer JP (1995) Increased collateral sprouting of primary afferents in the myelin-free spinal cord. J Neurosci $15: 2756-2767$.
Slaughter T, Wang J, Black MM (1997) Microtubule transport from the cell body into the axons of growing neurons. J Neurosci 17:5807-5819.

Smith CL (1994) The initiation of neurite outgrowth by sympathetic neurons grown in vitro does not depend on assembly of microtubules. J Cell Biol 127:1407-1418.

Szebenyi G, Callaway JL, Dent EK, Kalil K (1998) Interstitial branches develop from active regions of the axon demarcated by the primary growth cone during pausing behaviors. J Neurosci 18:7930-7940.

Tanaka E, Ho T, Kirschner MW (1995) The role of microtubule dynamics in growth cone motility and axon growth. J Cell Biol 128:139-155.

Terasaki M, Schmidek A, Galbraith JA, Gallant PE, Reese TS (1995) Transport of cytoskeletal elements in the squid giant axon. Proc Natl Acad Sci USA 92:11500-11503.

Vasquez RJ, Howell B, Yvon AC, Wadsworth P, Cassimeris L (1997) Nanomolar concentrations of nocodazole alter microtubule dynamic instability in vivo and in vitro. Mol Biol Cell 8:973-985.

Williamson T, Gordon-Weeks PR, Schachner M, Taylor J (1996) Microtubule reorganization is obligatory for growth cone turning. Proc Natl Acad Sci USA 93:15221-15226.

Witte S, Stier H, Cline HT (1996) In vivo observations of timecourse and distribution of morphological dynamics in Xenopus retinotectal axon arbors. J Neurobiol 31:219-234

Yu W, Baas PW (1995) The growth of the axon is not dependent upon net microtubule assembly at its distal tip. J Neurosci 15:6627-6833.

Yu W, Ahmad FJ, Baas PW (1994) Microtubule fragmentation and partitioning during axon collateral branch formation. J Neurosci 14:5872-5884.

Yu W, Schwei MJ, Baas PW (1996) Microtubule transport and assembly during axon growth. J Cell Biol 133:151-157.

Zhang L, Schmidt RE, Yan Q, Snider WD (1994) NGF and NT-3 have differing effects on the growth of dorsal root axons in developing mammalian spinal cord. J Neurosci 14:5187-5201. 